\title{
The Bacteria Genome Pipeline (BAGEP): An automated, scalable workflow for bacteria genomes with Snakemake
}

\author{
Idowu B Olawoye ${ }^{\text {Corresp., 1, }}$, Simon D.W. Frost ${ }^{3,4}$, Christian T Happi ${ }^{\text {Corresp. 1, } 2}$ \\ 1 Department of Biological Sciences, Faculty of Natural Sciences, Redeemer's University, Ede, Osun State, Nigeria \\ African Centre of Excellence for Genomics of Infectious Diseases (ACEGID), Redeemer's University, Ede, Osun State, Nigeria \\ 3 Microsoft Research, Redmond, Washington, USA \\ 4 London School of Hygiene \& Tropical Medicine, University of London, London, London, United Kingdom \\ Corresponding Authors: Idowu B Olawoye, Christian T Happi \\ Email address: olawoyei0303@run.edu.ng, happic@run.edu.ng
}

Next generation sequencing technologies are becoming more accessible and affordable over the years, with entire genome sequences of several pathogens being deciphered in few hours. However, there is the need to analyze multiple genomes within a short time, in order to provide critical information about a pathogen of interest such as drug resistance, mutations and genetic relationship of isolates in an outbreak setting. Many pipelines that currently do this are stand-alone workflows and require huge computational requirements to analyze multiple genomes. We present an automated and scalable pipeline called BAGEP for monomorphic bacteria that performs quality control on FASTQ paired end files, scan reads for contaminants using a taxonomic classifier, maps reads to a reference genome of choice for variant detection, detects antimicrobial resistant (AMR) genes, constructs a phylogenetic tree from core genome alignments and provide interactive short nucleotide polymorphism (SNP) visualization across core genomes in the data set. The objective of our research was to create an easy to use pipeline from existing bioinformatics tools that can be deployed on a personal computer. The pipeline was built on Snakemake framework and utilizes existing tools for each processing step: fastp for quality trimming, snippy for variant calling, Centrifuge for taxonomic classification, Abricate for AMR gene detection, snippy-core for generating whole and core genome alignments, IQ-TREE for phylogenetic tree construction and vcfR for an interactive heatmap visualization which shows SNPs at specific locations across the genomes. BAGEP was successfully tested and validated with Mycobacterium tuberculosis $(n=20)$ and Salmonella enterica serovar Typhi $(n=20)$ genomes which are about 4.4 million and 4.8 million base pairs, respectively. Running these test data on a 8 GB RAM, $2.5 \mathrm{GHz}$ quad core laptop took 122 and 61 minutes on respective data sets to complete the analysis. BAGEP is a fast, calls accurate SNPs and an easy to run pipeline that can be executed on a mid-range laptop; it is freely available on: https://github.com/idolawoye/BAGEP . 
1 The Bacteria Genome Pipeline (BAGEP): An

2 automated, scalable workflow for bacteria genomes

3 with Snakemake.

4

5

6

7

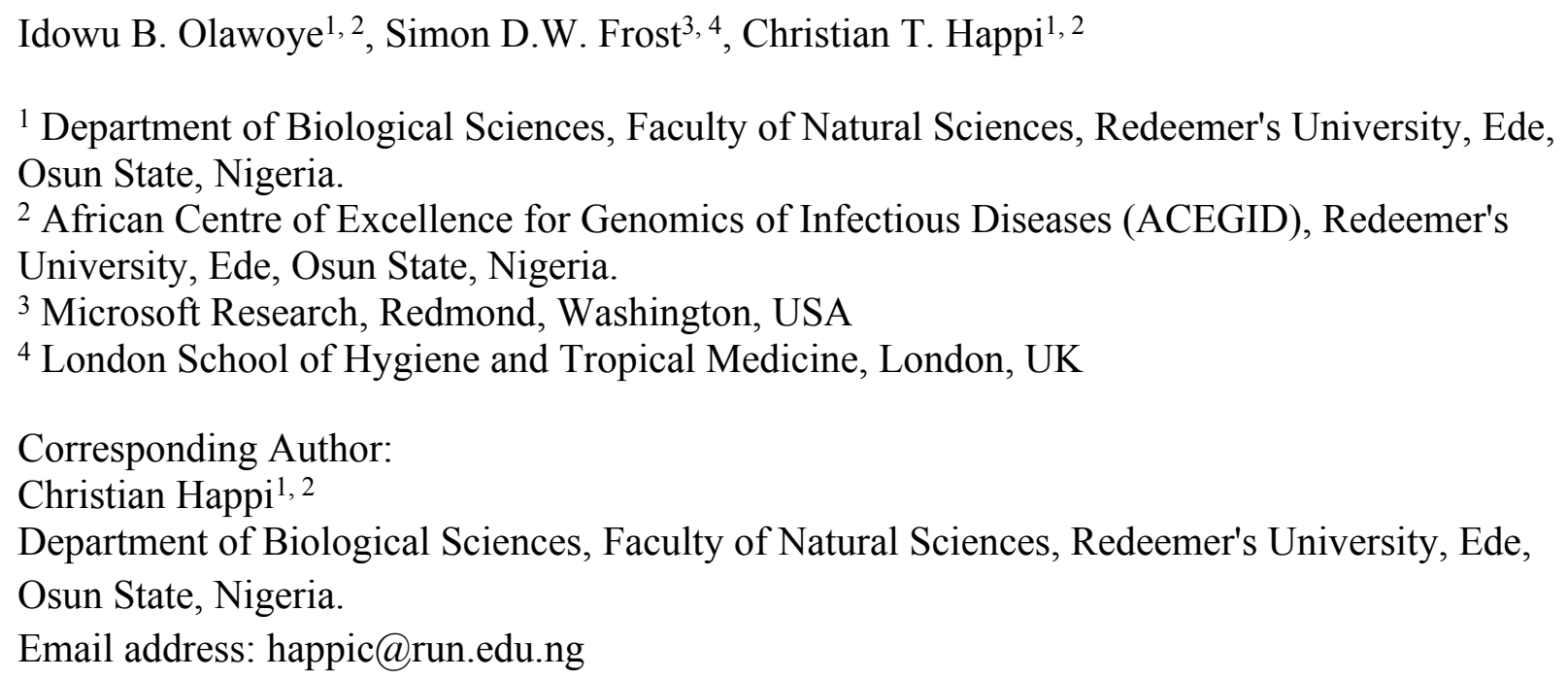




\section{Abstract}

47 Next generation sequencing technologies are becoming more accessible and affordable over the 48 years, with entire genome sequences of several pathogens being deciphered in few hours. 49 However, there is the need to analyze multiple genomes within a short time, in order to provide 50 critical information about a pathogen of interest such as drug resistance, mutations and genetic 51 relationship of isolates in an outbreak setting. Many pipelines that currently do this are stand52 alone workflows and require huge computational requirements to analyze multiple genomes. We 53 present an automated and scalable pipeline called BAGEP for monomorphic bacteria that 54 performs quality control on FASTQ paired end files, scan reads for contaminants using a 55 taxonomic classifier, maps reads to a reference genome of choice for variant detection, detects 56 antimicrobial resistant (AMR) genes, constructs a phylogenetic tree from core genome 57 alignments and provide interactive short nucleotide polymorphism (SNP) visualization across core genomes in the data set. The objective of our research was to create an easy to use pipeline 59 from existing bioinformatics tools that can be deployed on a personal computer.

60 The pipeline was built on Snakemake framework and utilizes existing tools for each processing 61 step: fastp for quality trimming, snippy for variant calling, Centrifuge for taxonomic 62 classification, Abricate for AMR gene detection, snippy-core for generating whole and core 63 genome alignments, IQ-TREE for phylogenetic tree construction and vcfR for an interactive 64 heatmap visualization which shows SNPs at specific locations across the genomes. BAGEP was 65 successfully tested and validated with Mycobacterium tuberculosis $(\mathrm{n}=20)$ and Salmonella 66 enterica serovar Typhi $(\mathrm{n}=20)$ genomes which are about 4.4 million and 4.8 million base pairs, 67 respectively. Running these test data on a 8 GB RAM, $2.5 \mathrm{GHz}$ quad core laptop took 122 and 6861 minutes on respective data sets to complete the analysis. BAGEP is a fast, calls accurate SNPs 
69 and an easy to run pipeline that can be executed on a mid-range laptop; it is freely available on:

70 https://github.com/idolawoye/BAGEP.

\section{Introduction}

72 Over the years, as next generation sequencing has rapidly become popular, molecular biology

73 has taken a giant leap in the way genomes of various organisms are deciphered. Genomics have

74 grown expeditiously with high throughput sequencing technologies and paving the way for novel

75 biological analytical approaches (Schuster, 2008) $\square$.

76 Genome sequencing of pathogens such as bacteria and viruses generate FASTQ files which

77 contains quality cores of the nucleotide bases in raw format. These files usually need various

78 technical processing steps such as adapter removal, sequence quality filtering and quality control.

79 This is crucial for other downstream analysis, as seen in a work where choosing the appropriate

80 FASTQ pre-processor improved downstream analysis significantly in comparison to other

81 software (Chen et al., 2018) $\square$.

82 In addition, reconstructing a sequenced genome is usually achieved through de novo assembly

83 (genome assembly using overlapping regions in the genome) or through a reference sequence

84 guided approach (mapping sequence reads to a reference genome) (Korbel et al., 2007; Zhang et

85 al., 2011) $\square$. There are numerous tools that can perform these tasks at various efficiency and for

86 different lengths of sequence reads. A few genome assemblers such as SPAdes, Burrows-

87 Wheeler Alignment tool (BWA), minimap2 and Bowtie are widely used in bioinformatics

88 (Bankevich et al., 2012; Langmead and Salzberg, 2012; Li, 2018; Li and Durbin, 2009) $\square$.

89 Variants in microbes occur as short nucleotide polymorphisms (SNPs), insertions and deletions

90 (indels) and or translocations. Mapping the reads to a reference genome or comparing the

91 assembled genome to a reference genome are the ways in detecting variants (Olson et al., 
92 2015) $\square$. SNP detection is important for comparative genomics in bacterial isolates as they have

93 been used to characterized different bacteria species sharing the same genus (Ledwaba et al.,

94 2019) $\square$. SNP analysis is also an integral part of understanding evolution in bacterial genomes,

95 detecting the cause or source of an outbreak, phylogeography and genome-wide association

96 studies (GWAS) (Farhat et al., 2019; O’Neill et al., 2019; Stimson et al., 2019) $\square$.

97 At the moment, there is no generally accepted standard operating procedure for evaluating and

98 calling SNPs and or indels which has led to a wide variety of tools and methods for variant

99 detection. Furthermore, best practices for variant identification in microbial genomes have been

100 proposed in literature and it has been adopted to a large extent (Olson et al., 2015; Van der

101 Auwera et al., 2013) $\square$.

102 Downstream analysis of numerous bacteria genomes using whole genome sequencing (WGS)

103 pipelines require high performance computing servers or a cloud-based support system. In

104 addition, while a number of WGS pipeline exist such as UVP (Ezewudo et al., 2018) and

105 MTBseq (Kohl et al., 2018) for Mycobacterium tuberculosis, these pipelines require huge

106 computing infrastructure. Moreover, the pipelines usually have many dependencies to be

107 installed especially if the analysis requires multiple tasks to be performed such as phylogenetic

108 tree construction, drug resistance prediction, clustering, and so on. The huge amount of

109 bioinformatics tools available makes it a daunting task in picking the suitable software for a

110 certain analysis. In low-to-middle countries who are becoming exposed to WGS of pathogens, it

111 is important to also provide ready-to-use, quick and easily deployable pipelines for WGS data

112 analysis. These pipelines should also be a stand-alone workflow on a local server or even a

113 personal computer. For example, UVP and MTBseq needs at least 100 GB and 25 GB of RAM 
114 respectively to run locally which is capitally intensive for many researchers in low-to-middle

115 countries (LMICs) who need to analyze WGS data.

116 To address the challenges identified above, we embarked on the development of BAGEP

117 (Bacteria Genome Pipeline, available online at https://github.com/idolawoye/BAGEP\#). It uses

118 existing pipelines and bioinformatics tools and an advanced workflow management system

119 called Snakemake (Koster and Rahmann, 2012) $\square$. This pipeline combines many tools used in

120 downstream analysis of paired-end FASTQ raw reads such as quality control, read mapping,

121 variant calling, core genome and/or full genome alignments, SNP visualization and phylogeny

122 into a single, fast, easy to use workflow.

\section{Materials \& Methods}

\section{Sampling}

125 To display the versatility of BAGEP, we applied it to a set of multi-drug resistant (MDR) $M$.

126 tuberculosis genomes (n=20) from Southwest Nigeria (Senghore et al., 2017) $\square$ and S. enterica

127 serovar Typhi genomes (n=20) from the United Kingdom (Ashton et al., 2017) $\square$. These publicly

128 available paired-end FASTQ reads were extracted from National Centre for Biotechnology

129 Information (NCBI) Sequence Read Archive (SRA). Genomic DNA of isolates were extracted

130 from liquid cultures and sequenced on Illumina MiSeq and HiSeq platforms. These raw reads are

131 deposited under BioProjects PRJEB15857 and PRJNA248792.

\section{Implementation}

133 Installation of BAGEP requires the pipeline to be downloaded on to a personal computer and

134 installed by creating a conda environment to set up all dependencies. Centrifuge database should

135 be downloaded separately in order to detect contaminants in the samples. Complete installation 
136 steps are stated in the github README file:

137 https://github.com/idolawoye/BAGEP/blob/master/README.md.

138 The analysis of BAGEP is segmented into set of 'rules' that connects the output file of an

139 analysis into the input of the next task in the general workflow (Figure 1). The dependencies are

140 fastp for quality control of raw reads, Centrifuge for taxonomic classification, Snippy for variant

141 detection, snippy-core for core and whole genome alignments, Abricate for AMR detection, IQ-

142 TREE for phylogenetics, Krona for taxonomic visualization, vcfR and heatmaply for SNP

143 visualization.

144 These tools can be installed in a containerized manner using a bioconda channel (Dale et al.,

145 2018) $\square$ which can be activated and deactivated easily, whereas the SNP visualization is provided

146 by R libraries included in the dependencies. The input files for BAGEP are paired-end FASTQ

147 files and a reference genome in FASTA or GenBank format, only the latter is provided in the

148 single line command to run, whilst the former should be saved in a designated local directory.

149 BAGEP also allows full customization of the pipeline, such that users can modify the parameters

150 used in running their samples. For example, the pipeline does not mask repeat regions when

151 aligning the core genomes by default, but this can be done by adding the option to the snakefile

152 rule handling that process. It is possible to modify every step of the workflow to suit the samples

153 being processed, even the model for phylogenetics.

154 Upon execution of the pipeline, Snakemake organizes the correct combination of tasks in order

155 to generate the desired output and also leverages on the wildcard feature to analyze multiple

156 genomes that have similar format allowed in the rule (Koster and Rahmann, 2012) $\square$. Preliminary

157 results such as quality trimmed FASTQ files are saved in a new directory, while primary results

158 like detected variants, core genome alignments, phylogeny and SNP visualization are saved in a 
159 different directory. The pipeline runs on Linux/Unix, MacOS terminal or Windows Subsystem

160 for Linux. However, no prior skill in programming is required to run and interpret the results as

161 only one stage of input is needed and thereafter the pipeline runs automatically. Another

162 significant advantage of BAGEP is that it can re-execute steps that have not been ran if errors

163 occur and resume from where it stopped rather than restarting the entire process which another

164 valuable feature of the underlying framework.

165 Results

166 FASTQ Pre-processing

167 BAGEP performs several quality control tasks on raw FASTQ reads using fastp (Chen et al., 168 2018) $\square$. Fastp capitalizes on speed, efficiency and an all-in-one pre-processor. It carries out 169 sliding window quality filtering, adapter trimming, base correction, polyG and polyX tail

170 trimming for Illumina NextSeq and NovaSeq series, UMI pre-processing to reduce background

171 noise and improve sensitivity when detecting ultra-low frequency mutations in deep sequencing,

172 duplication analysis, quality control and reporting (Figure 2).

173 Fastp was chosen as the suitable tool for this task as it is seen to outperform other quality control

174 FASTQ software and it also allows multi-threading to improve its performance when dealing 175 with numerous samples (Chen et al., 2018) $\square$.

\section{Taxonomic classification}

177 This step is crucial in the pipeline as contaminants from the environment such as host cells or 178 other bacteria can negatively impact the accuracy of variants detected. We utilized Centrifuge for 179 this step due to its lower memory requirements compared to other similar tools like Kraken (Kim 180 et al., 2016) $\square$. To visualize the results, Krona was employed such that one can interact with the 181 output and also export it as a static image file (Figure 3) (Ondov et al., 2011) $\square$. 


\section{Read mapping and variant discovery}

183 Mapping refined sequence reads to the reference genome is done by Snippy (Seemann, 2015) $\square$

184 which is a variant calling and core genome alignment pipeline. Firstly, BWA MEM (Li and

185 Durbin, 2009) $\square$ maps reads to the provided reference genome and they are manipulated with

186 Samtools (Li et al., 2009) $\square$. Variants are called from the resulting Binary Alignment Map

187 (BAM) files using freebayes (Garrison and Marth, 2012) $\square$ which takes short-read alignments in

188 BAM formats and Phred +33 quality scores from each sample and determines the best

189 combination of polymorphisms in each sample at each position in the reference genome with the

190 aid of Bayesian variant detection model. The variants are annotated with SnpEff (Cingolani et

191 al., 2012) $\square$, with reports generated in variant call format (VCF), TAB, CSV, HTML, BED, GFF

192 and FASTA files. A unified VCF file that contains core and full genome alignments of individual

193 SNP output files are generated with Snippy-core. Furthermore, BAGEP leverages on a function

194 in Snippy-core that allows the user to filter out problematic or repetitive regions core genomes of

195 the samples such as Proline-Glutamate (PE) and Proline-Proline-Glutamate (PPE) gene families

196 in M. tuberculosis by providing a BED file to omit these regions.

197 Antimicrobial resistant gene screening

198 BAGEP uses abricate to screen the isolates for antimicrobial resistant (AMR) genes by using

199 either one of the many AMR databases that comes with it. The great thing about abricate is that

200 these databases can be updated from the help option and the user can select which databases suits

201 their analysis. The results from this step is stored in a tab-separated file which can be viewed in a

202 variety of software.

203 SNP visualisation 
204 One other key feature of BAGEP is the ability to generate an interactive SNP visualization that 205 allows the end user to view substitution polymorphisms across core genomes in the population.

206 In the pipeline, a custom R script makes use of vcfR (Knaus and Grünwald, 2017) $\square$ and

207 heatmaply (Galili et al., 2017) $\square$, parses the VCF file output from Snippy-core to render a

208 heatmap showing SNP positions in a HTML file (Figure 4).

209 The heatmap can be interacted with, with a number of tools such as zooming, hovering over the 210 image to show SNPs and export a selected region as an image. This is very useful in a VCF file 211 with high number of samples and reported SNPs as hovering around regions in the image can 212 infer which position the polymorphism occur and in what sample.

\section{Phylogeny}

214 The final analysis done by BAGEP is the construction of a maximum-likelihood phylogenetic 215 tree with IQ-TREE (Nguyen et al., 2015) $\square$, this step uses the core genome alignment generated 216 by Snippy-core with ultra-fast bootstrap value of 1000 . The output tree is deposited in the 217 'results' directory and can be viewed and annotated with any tree viewing software such as 218 Figtree or Interactive Tree of Life (ITOL).

\section{Performance}

220 Running BAGEP on 20 M. tuberculosis and 20 S. enterica serovar Typhi genomes with an 8 GB

221 RAM, $2.5 \mathrm{GHz}$ quad core laptop took 122 and 61 minutes to complete the analysis, respectively; 222 M. tuberculosis has a 4.4 million base pair genome while $S$. enterica serovar Typhi has 4.81 223 million base pairs. BAGEP capitalizes on Snakemake's multi-threading feature which implies 224 that it can be deployed on laptops with greater performance or a computing server to improve its 225 speed. Comparing BAGEP's performance to Nullarbor pipeline on a benchmark computer using 226 the same M. tuberculosis data sets, BAGEP took 122 minutes whilst Nullarbor spent 186 minutes 
227 to complete the same analysis. In addition to speed, BAGEP outperforms Nullarbor in detecting

228 more accurate SNPs. To assess the robustness of BAGEP, we ran the pipeline on $57 \mathrm{M}$.

229 tuberculosis genomes using a 16 GB RAM computer and it completed the run in 225 minutes

230 (Supplementary file 1).

\section{Discussion}

232 BAGEP was built around the core concepts of Snakemake which offers parallelization of several 233 tasks in an orderly manner. It was designed to be user friendly, fast, customizable and 234 reproducible. In addition, we wanted a pipeline that can be deployed on a personal computer and 235 handle medium to large data and finally, an interactive tool for visualizing SNPs that were 236 detected whilst running the pipeline. BAGEP requires minimal user input and runs from start to 237 finish with a single command.

238 As the backbone of BAGEP is Snakemake, each rule is run in its own environment and allows 239 the combination of other programming languages such as Python, R and Bash. This also implies 240 that each rule can be customized to make it run faster depending on the configuration of the 241 machine running it and basic understanding of tools used in the pipeline. For example, the rule 242 that takes the longest time to run is the step where reads are mapped to a reference genome and 243 variants are called. This is executed by Snippy and the number of threads can be increased to 244 speed up the process.

245 This pipeline has been tested with M. tuberculosis and S. enterica serovar Typhi and it is suitable 246 for genetically monomorphic or monoclonal pathogens such as Yersinia pestis, Bacillus

247 anthracis, Mycobacterium leprae and Treponema pallidum due to the limited amount of 248 variations in their core genome as compared to highly recombinant pathogens (Achtman, $2492008) \square$. 
250 For visualization, BAGEP generates a HTML file as output that contains observed SNPs in core

251 genomes of the samples and the position where they can be found, in addition with dendrograms

252 that highlight the relatedness of the genomes. The interactive image can be zoomed in and out,

253 reveal SNP regions by hovering around the image with a mouse and also export the image as

254 portable network graphics (PNG) image. This image can be easily interpreted by anyone with

255 little or no knowledge in bioinformatics and gives a summary of the analysis.

256 It is worthy to note that BAGEP was compiled with speed in mind and ease-of-use and that is

257 why the dependencies can be installed in a conda environment under the bioconda channel (Dale

258 et al., 2018) $\square$. Performance comparison to Nullarbor shows that BAGEP identified 459 SNPs in

259 a particular isolate which yielded 598 SNPs with Nullarbor (it is important to note that BAGEP

260 and Nullarbor uses the same tool, Snippy for variant detection). In other to validate the accuracy

261 of the SNPs, we ran the same sample as raw reads (that is without undergoing QC steps with

262 fastp or Trimmomatic) and the end result was 598 SNPs, this trend was seen in other samples as

263 well. This indicates that the QC step in Nullarbor is not as effective as the one embedded in

264 BAGEP, which will give rise to false positives when using Nullarbor. Furthermore, BAGEP is

265 quicker than Nullarbor when analyzing the same set of samples. Therefore, standard population

266 genomics of low recombinant or genetically monomorphic bacteria can be conducted with

267 BAGEP as it handles the fundamental analysis needed.

\section{Conclusions}

269 We present BAGEP, a fully automated and scalable pipeline that is built on Snakemake

270 framework. This pipeline will be useful for researchers in low-to-middle income countries and

271 people with little or no bioinformatics skills in analyzing raw genomics data. 
272 It is effective for running medium to large data sets of paired-end raw reads of bacteria genomes,

273 and has been tested with M. tuberculosis and S. enterica serovar Typhi. We have also shown that

274 it is suitable for genetically monomorphic or monoclonal pathogens such as Yersinia pestis,

275 Bacillus anthracis, Mycobacterium leprae and Treponema pallidum. Some of BAGEP's

276 advantages is that it is quick, identifies SNPs with better accuracy, easy to run and can be

277 deployed on a mid-range laptop computer. Our future plan is to improve this pipeline by adding

278 new features and roll out updates as we collaborate with other scientists. We believe this will be

279 useful for researchers in low-to-middle income countries and people with little or no

280 bioinformatics skills in analyzing raw genomics data.

\section{Acknowledgements}

282 We will like to appreciate the entire team at the African Center of Excellence of Genomics for 283 Infectious Diseases (ACEGID), Redeemer's University, Nigeria and the Lab of Viral Zoonotics

284 (LVZ), Department of Veterinary Medicine, University of Cambridge, UK for establishing the 285 partnership that birthed the idea behind BAGEP.

\section{Data Availability}

287 BAGEP source code and documentation is freely available on GitHub at: 288 https:/github.com/idolawoye/BAGEP. The Salmonalla enetrica serova Typhi test data used can 289 be found at: http://doi.org/10.5281/zenodo.3731118

\section{References}

291

292

293

294

295

296
Achtman, M. (2008). Evolution, Population Structure, and Phylogeography of Genetically Monomorphic Bacterial Pathogens. Annual Review of Microbiology, 62(1), 53-70. https://doi.org/10.1146/annurev.micro.62.081307.162832

Ashton, P. M., Owen, S. V., Kaindama, L., Rowe, W. P. M., Lane, C. R., Larkin, L., Nair, S., Jenkins, C., de Pinna, E. M., Feasey, N. A., Hinton, J. C. D., and Dallman, T. J. (2017). Public health surveillance in the UK revolutionises our understanding of the invasive 
297

298

299

300

301

302

303

304

305

306

307

308

309

310

311

312

313

314

315

316

317

318

319

320

321

322

323

324

325

326

327

328

329

330

331

332

333

334

Salmonella Typhimurium epidemic in Africa. Genome Medicine, $9(1), 92$. https://doi.org/10.1186/s13073-017-0480-7

Bankevich, A., Nurk, S., Antipov, D., Gurevich, A. A., Dvorkin, M., Kulikov, A. S., Lesin, V. M., Nikolenko, S. I., Pham, S., Prjibelski, A. D., Pyshkin, A. V., Sirotkin, A. V., Vyahhi, N., Tesler, G., Alekseyev, M. A., and Pevzner, P. A. (2012). SPAdes: A new genome assembly algorithm and its applications to single-cell sequencing. Journal of Computational Biology, 19(5), 455-477. https://doi.org/10.1089/cmb.2012.0021

Chen, S., Zhou, Y., Chen, Y., and Gu, J. (2018). Fastp: An ultra-fast all-in-one FASTQ preprocessor. Bioinformatics. https://doi.org/10.1093/bioinformatics/bty560

Cingolani, P., Platts, A., Wang, L. L., Coon, M., Nguyen, T., Wang, L., Land, S. J., Lu, X., and Ruden, D. M. (2012). A program for annotating and predicting the effects of single nucleotide polymorphisms, SnpEff: SNPs in the genome of Drosophila melanogaster strain w1118; iso-2; iso-3. Fly, 6(2), 80-92. https://doi.org/10.4161/fly.19695

Dale, R., Grüning, B., Sjödin, A., Rowe, J., Chapman, B. A., Tomkins-Tinch, C. H., Valieris, R., Batut, B., Caprez, A., Cokelaer, T., Yusuf, D., Beauchamp, K. A., Brinda, K., Wollmann, T., Corguillé, G. Le, Ryan, D., Bretaudeau, A., Hoogstrate, Y., Pedersen, B. S., ... Köster, J. (2018). Bioconda: Sustainable and comprehensive software distribution for the life sciences. Nature Methods, 15(7), 475-476. https://doi.org/10.1038/s41592-018-0046-7

Ezewudo, M., Borens, A., Chiner-Oms, Á., Miotto, P., Chindelevitch, L., Starks, A. M., Hanna, D., Liwski, R., Zignol, M., Gilpin, C., Niemann, S., Kohl, T. A., Warren, R. M., Crook, D., Gagneux, S., Hoffner, S., Rodrigues, C., Comas, I., Engelthaler, D. M., Alland, D., Rigouts, L., Lange, C., Dheda, K., Hasan, R., McNerney, R., Cirillo, D. M., Marco Schito, Rodwell, T. C., Posey, J. (2018). Integrating standardized whole genome sequence analysis with a global Mycobacterium tuberculosis antibiotic resistance knowledgebase. Scientific Reports, 8(1), 1-10. https://doi.org/10.1038/s41598-018-33731-1

Galili, T., O’Callaghan, A., Sidi, J., and Sievert, C. (2017). heatmaply: an R package for creating interactive cluster heatmaps for online publishing. Bioinformatics, 34(9), 1600-1602. https://doi.org/10.1093/bioinformatics/btx657

Garrison, E., and Marth, G. (2012). Haplotype-based variant detection from short-read sequencing. ArXiv Preprint ArXiv:1207.3907.

Hendriksen, R. S., Price, L. B., Schupp, J. M., Gillece, J. D., Kaas, R. S., Engelthaler, D. M., Bortolaia, V., Pearson, T., Waters, A. E., Upadhyay, B. P., Shrestha, S. D., Adhikari, S., Shaky, G., Keim, P. S., and Aarestrupa, F. M. (2011). Population genetics of vibrio cholerae from Nepal in 2010: Evidence on the origin of the haitian outbreak. MBio, 2(4). https://doi.org/10.1128/mBio.00157-11

Keim, P. S., and Wagner, D. M. (2009). Humans and evolutionary and ecological forces shaped the phylogeography of recently emerged diseases. In Nature Reviews Microbiology (Vol. 7, Issue 11, pp. 813-821). Nature Publishing Group. https://doi.org/10.1038/nrmicro2219 
335

336

337

338

339

340

341

342

343

344

345

346

347

348

349

350

351

352

353

354

355

356

357

358

359

360

361

362

363

364

365

366

367

368

369

370

371

372

Kim, D., Song, L., Breitwieser, F. P., \& Salzberg, S. L. (2016). Centrifuge: rapid and sensitive classification of metagenomic sequences. Genome research, 26(12), 1721-1729. https://doi.org/10.1101/gr.210641.116

Knaus, B. J., and Grünwald, N. J. (2017). vcfr: a package to manipulate and visualize variant call format data in R. Molecular Ecology Resources, 17(1), 44-53. https://doi.org/10.1111/1755-0998.12549

Kohl, T. A., Utpatel, C., Schleusener, V., De Filippo, M. R., Beckert, P., Cirillo, D. M., and Niemann, S. (2018). MTBseq: A comprehensive pipeline for whole genome sequence analysis of Mycobacterium tuberculosis complex isolates. PeerJ, 2018(11). https://doi.org/10.7717/peerj.5895

Korbel, J. O., Urban, A. E., Affourtit, J. P., Godwin, B., Grubert, F., Simons, J. F., Kim, P. M., Palejev, D., Carriero, N. J., Du, L., Taillon, B. E., Chen, Z., Tanzer, A., Saunders, A. C. E., Chi, J., Yang, F., Carter, N. P., Hurles, M. E., Weissman, S. M., Harkins, T. T., Gerstein, M.B., Egholm, M., Snyder, M. (2007). Paired-end mapping reveals extensive structural variation in the human genome. Science, 318(5849), 420-426.

https://doi.org/10.1126/science.1149504

Koster, J., and Rahmann, S. (2012). Snakemake--a scalable bioinformatics workflow engine. Bioinformatics, 28(19), 2520-2522. https://doi.org/10.1093/bioinformatics/bts480

Langmead, B., and Salzberg, S. L. (2012). Fast gapped-read alignment with Bowtie 2. Nature Methods, 9(4), 357-359. https://doi.org/10.1038/nmeth.1923

Li, H. (2018). Minimap2: Pairwise alignment for nucleotide sequences. Bioinformatics, 34(18), 3094-3100. https://doi.org/10.1093/bioinformatics/bty191

Li, H., and Durbin, R. (2009). Fast and accurate short read alignment with Burrows-Wheeler transform. Bioinformatics (Oxford, England), 25(14), 1754-1760. https://doi.org/10.1093/bioinformatics/btp324

Li, H., Handsaker, B., Wysoker, A., Fennell, T., Ruan, J., Homer, N., Marth, G., Abecasis, G., and Durbin, R. (2009). The sequence alignment/map format and SAMtools. Bioinformatics, 25(16), 2078-2079.

Nelson, C. L., Pelak, K., Podgoreanu, M. V., Ahn, S. H., Scott, W. K., Allen, A. S., Cowell, L. G., Rude, T. H., Zhang, Y., Tong, A., Ruffin, F., Sharma-Kuinkel, B. K., and Fowler, V. G. (2014). A genome-wide association study of variants associated with acquisition of Staphylococcus aureus bacteremia in a healthcare setting. BMC Infectious Diseases, 14(1), 83. https://doi.org/10.1186/1471-2334-14-83

Nguyen, L.-T., Schmidt, H. A., von Haeseler, A., and Minh, B. Q. (2015). IQ-TREE: a fast and effective stochastic algorithm for estimating maximum-likelihood phylogenies. Molecular Biology and Evolution, 32(1), 268-274. https://doi.org/10.1093/molbev/msu300

Olson, N. D., Lund, S. P., Colman, R. E., Foster, J. T., Sahl, J. W., Schupp, J. M., Keim, P., Morrow, J. B., Salit, M. L., and Zook, J. M. (2015). Best practices for evaluating single 
373

374

375

376

377

378

379

380

381

382

383

384

385

386

387

388

389

390

391

392

393

394

395

396

397

398

399

400

nucleotide variant calling methods for microbial genomics. In Frontiers in Genetics (Vol. 6, Issue JUL, p. 235). Frontiers Media S.A. https://doi.org/10.3389/fgene.2015.00235

Ondov, B.D., Bergman, N.H. \& Phillippy, A.M. Interactive metagenomic visualization in a Web browser. BMC Bioinformatics 12, 385 (2011). https://doi.org/10.1186/1471-2105-12-385

Sahl, J. W., Gillece, J. D., Schupp, J. M., Waddell, V. G., Driebe, E. M., Engelthaler, D. M., and Keim, P. (2013). Evolution of a Pathogen: A Comparative Genomics Analysis Identifies a Genetic Pathway to Pathogenesis in Acinetobacter. PLoS ONE, 8(1). https://doi.org/10.1371/journal.pone.0054287

Schuster, S. C. (2008). Next-generation sequencing transforms today's biology. In Nature Methods (Vol. 5, Issue 1, pp. 16-18). https://doi.org/10.1038/nmeth1156

Seemann, T. (2015). Snippy: Rapid haploid variant calling and core genome alignment. https://github.com/tseemann/snippy

Seemann T, Abricate, Github https://github.com/tseemann/abricate

Senghore, M., Otu, J., Witney, A., Gehre, F., Doughty, E. L., Kay, G. L., Butcher, P., Salako, K., Kehinde, A., Onyejepu, N., Idigbe, E., Corrah, T., De Jong, B., Pallen, M. J., and Antonio, M. (2017). Whole-genome sequencing illuminates the evolution and spread of multidrugresistant tuberculosis in Southwest Nigeria. PLoS ONE. https://doi.org/10.1371/journal.pone.0184510

Van der Auwera, G. A., Carneiro, M. O., Hartl, C., Poplin, R., del Angel, G., Levy-Moonshine, A., Jordan, T., Shakir, K., Roazen, D., Thibault, J., Banks, E., Garimella, K. V., Altshuler, D., Gabriel, S., and DePristo, M. A. (2013). From fastQ data to high-confidence variant calls: The genome analysis toolkit best practices pipeline. Current Protocols in Bioinformatics, 43(SUPL.43), 11.10.1-11.10.33. https://doi.org/10.1002/0471250953.bi1110s43

Zhang, W., Chen, J., Yang, Y., Tang, Y., Shang, J., and Shen, B. (2011). A Practical Comparison of De Novo Genome Assembly Software Tools for Next-Generation SequencingTechnologies. PLoS ONE, 6(3), e17915. https://doi.org/10.1371/journal.pone.0017915 


\section{Figure 1}

A schematic workflow of BAGEP.

Fastp is used for quality control on paired-end FASTQ reads. The processed reads are mapped against a reference genome provided by the user. Centrifuge is used to check the reads for contamination and generate a taxonomic visualized report with Krona. Variants are called using Freebayes and annotated with SnpEff (aided by Snippy). The resulting variant call format (VCF) files and genomes from each sample are collated with Snippy-core to produce a VCF file containing all samples, core and whole genome multiple sequence alignments. A maximum-likelihood phylogenetic tree is constructed with IQTREE and a HTML file containing an interactive SNP visualization with the VCF file. Finally, Abricate generates an AMR report from whole genome multiple sequence alignments. 


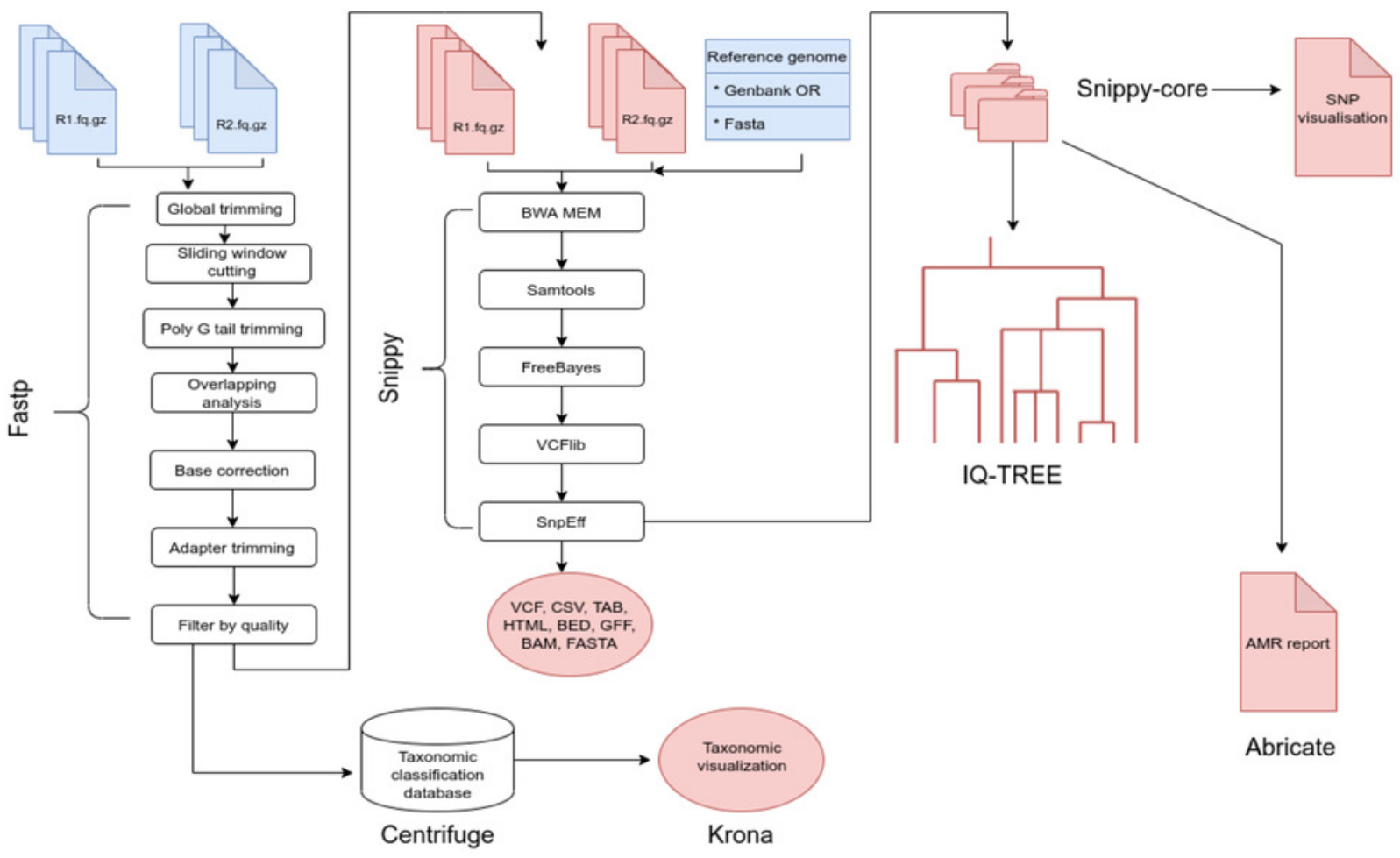


Figure 2

Pre-processing reports from read 1 of one of the M. tuberculosis genomes

(A) Read quality before filtering (B) Read quality after filtering (C) Base contents before filtering (D) Base contents after filtering.

Before filtering: read1: quality

A

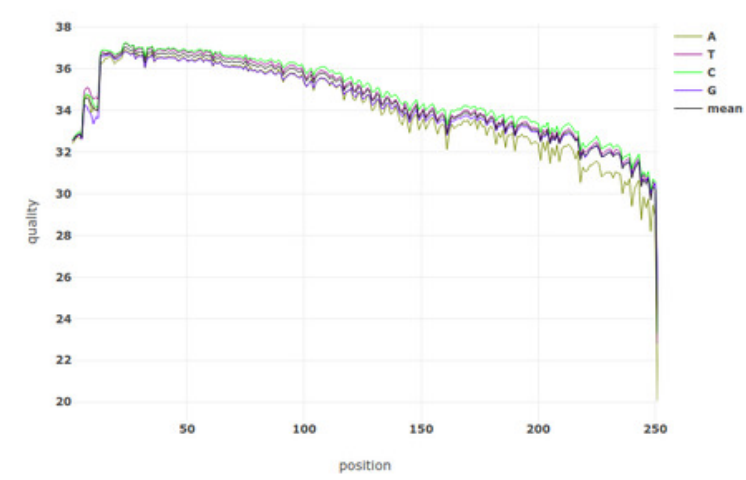

$\mathrm{C}$

Before filtering: read1: base contents
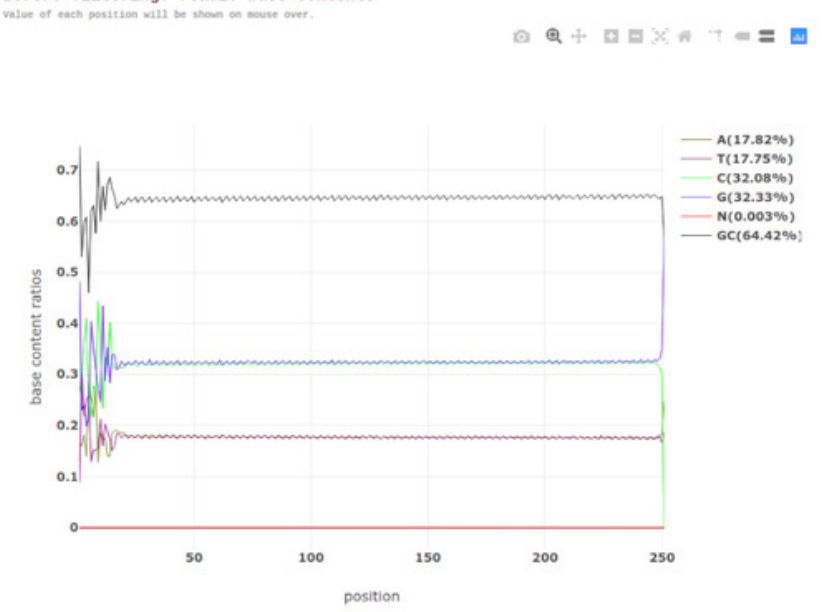

After filtering: read1: quality

B

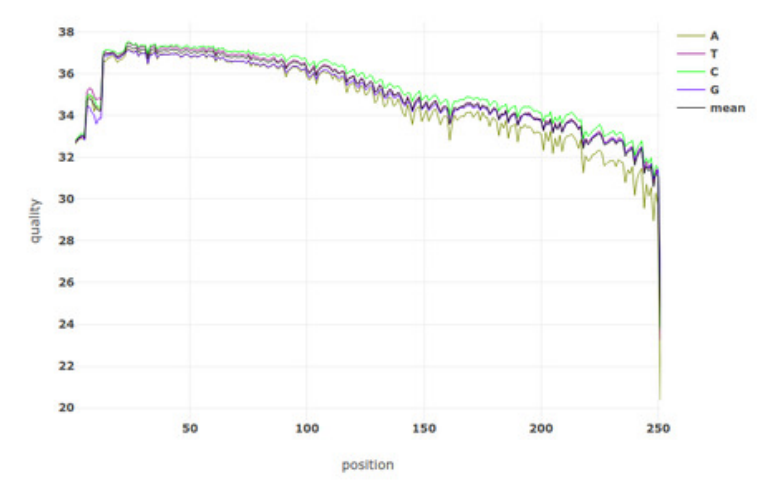

$\mathrm{D}$

After filtering: read1: base contents
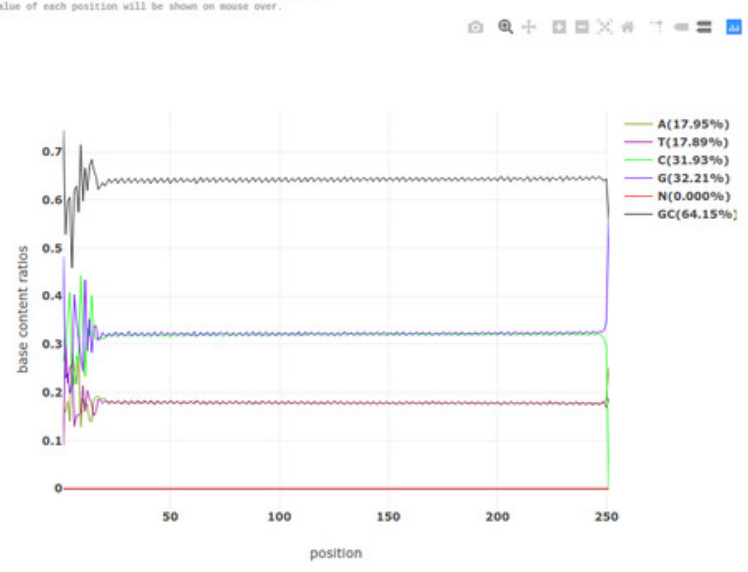
Figure 3

Taxonomic classification of reads in an isolate using Centrifuge and Krona

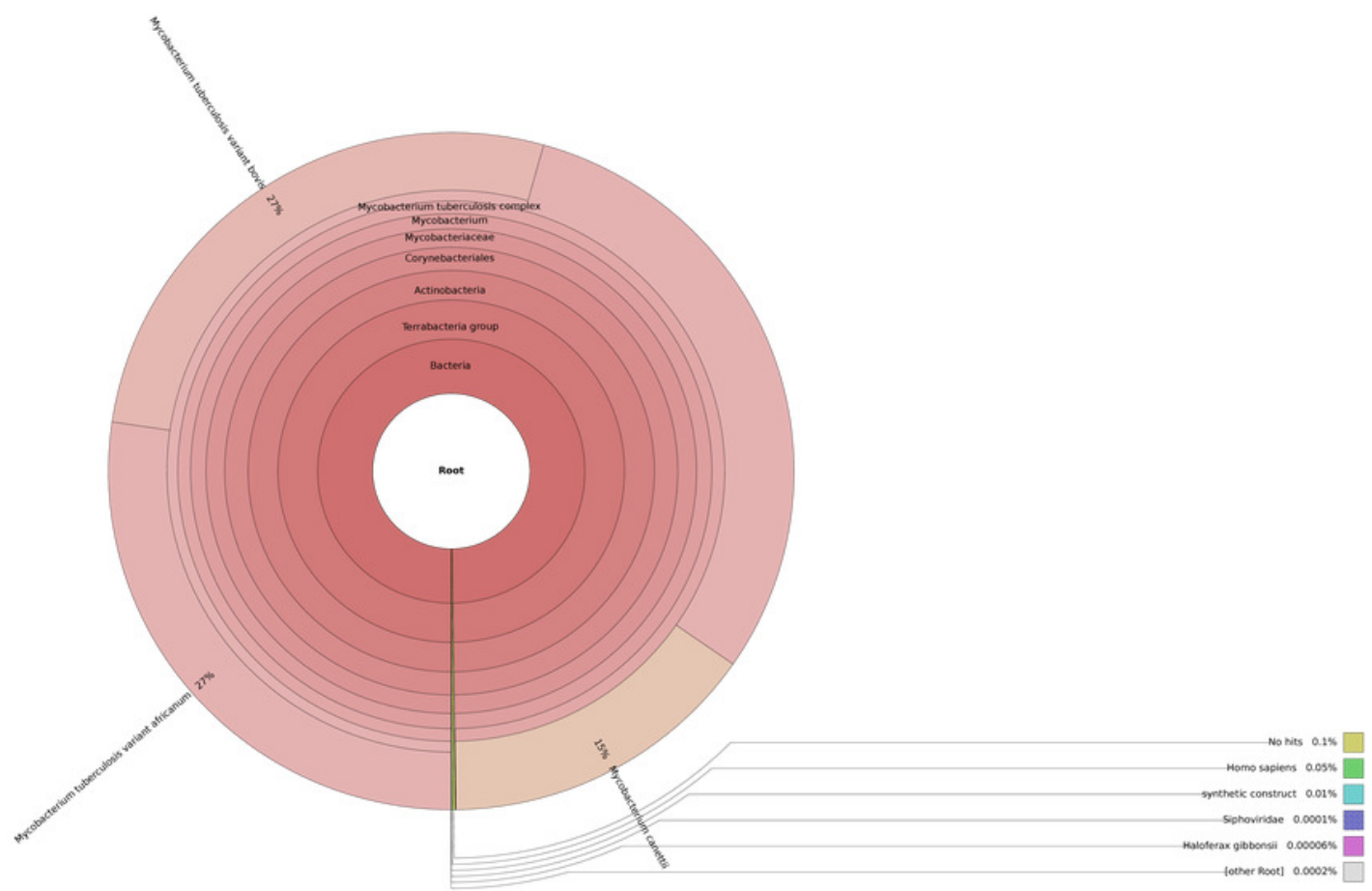




\section{Figure 4}

Interactive visualization of SNPs showing their positions across genomes

(A) Twenty (20) M. tuberculosis genomes (B) Twenty (20) Salmonella enterica serova Typhi genomes.
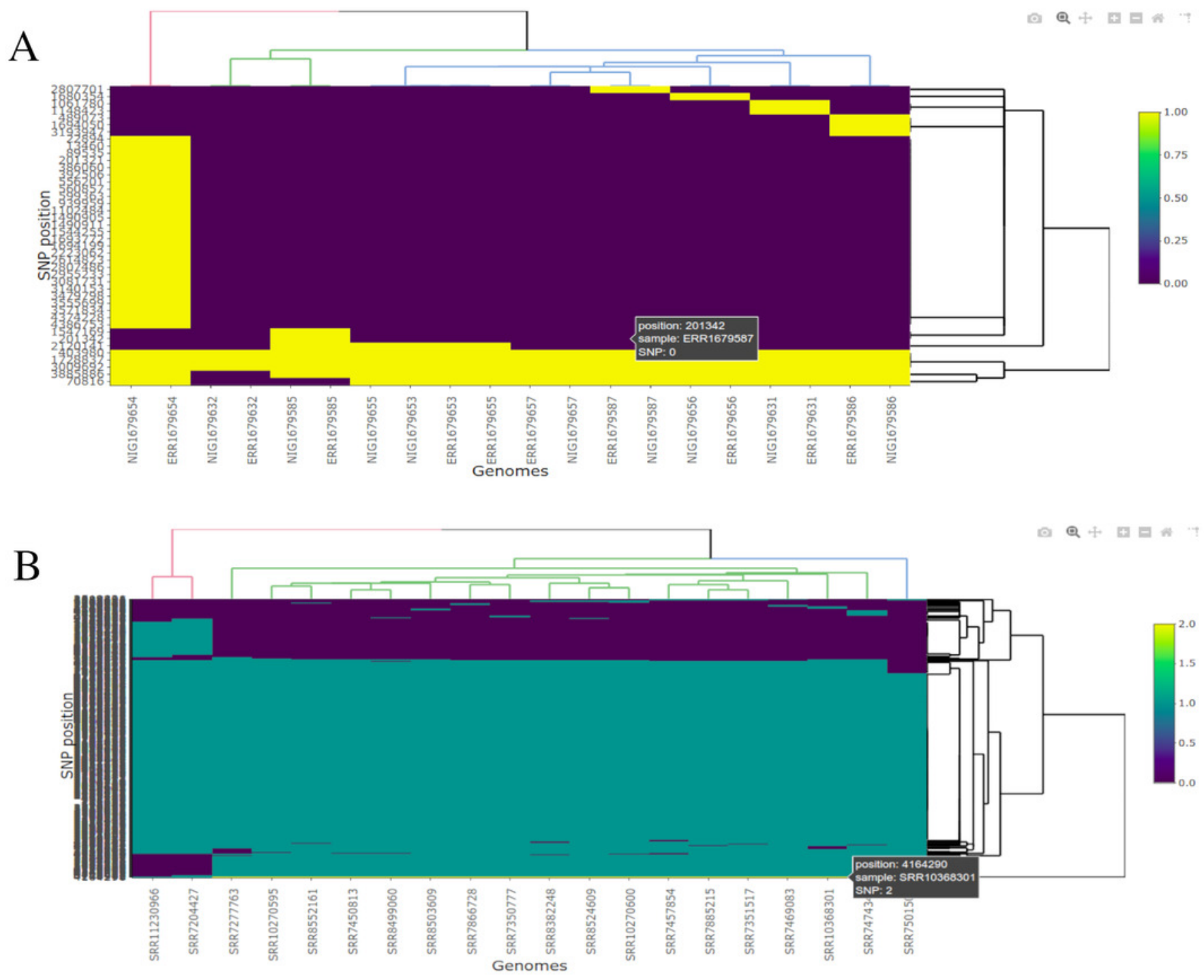